\title{
Comorbid Affective Symptomatology and Neurocognitive Performance in College Athletes
}

\author{
Garrett A. Thomas (D), Erin T. Guty (D), Kaitlin E. Riegler (D) and Peter A. Arnett (D) \\ Department of Psychology, The Pennsylvania State University, University Park, PA, USA
}

(Received November 2, 2020; Final Revision January 30, 2021; Accepted March 12, 2021; First Published Online May 5, 2021)

\begin{abstract}
Objectives: The current study aims to examine the prevalence rates and the relationship of symptoms of depression, anxiety, and comorbid depression/anxiety with neurocognitive performance in college athletes at baseline. We hypothesized a priori that the mood disturbance groups would perform worse than healthy controls, with the comorbid group performing worst overall. Methods: Eight hundred and thirty-one $(M=620, F=211)$ collegiate athletes completed a comprehensive neuropsychological test battery at baseline which included self-report measures of anxiety and depression. Athletes were separated into four groups [Healthy Control (HC) $(n=578)$, Depressive Symptoms Only $(n=137)$, Anxiety Symptoms Only $(n=54)$, and Comorbid Depressive/Anxiety Symptoms $(n=62)]$ based on their anxiety and depression scores. Athletes' neurocognitive functioning was analyzed via $Z$ score composites of Attention/ Processing Speed and Memory. Results: One-way analysis of variance revealed that, compared to HC athletes, the comorbid group performed significantly worse on measures of Attention/Processing Speed but not Memory. However, those in the depressive symptoms only and anxiety symptoms only groups were not significantly different from one another or the $\mathrm{HC}$ group on neurocognitive outcomes. Chi-square analyses revealed that a significantly greater proportion of athletes in all three affective groups were neurocognitively impaired compared to the HC group. Conclusions: These results demonstrate that collegiate athletes with comorbid depressive/anxiety symptoms should be identified, as their poorer cognitive performance at baseline could complicate post-concussion interpretation. Thus, assessing for mood disturbance at baseline is essential to obtain an accurate measurement of baseline functioning. Further, given the negative health outcomes associated with affective symptomatology, especially comorbidities, it is important to provide care as appropriate.
\end{abstract}

Keywords: Depression, Anxiety, Comorbidity, Concussion, Mild, Injury, Sports, Test, Neuropsychological

\section{INTRODUCTION}

Sports-related concussions (SRCs) are a common occurrence, with some research estimating between 1.6 and 3.8 million concussions related to sport and recreation occurring annually (Langlois et al., 2006). These numbers are far from static, however, as the NCAA Injury Surveillance Program reported a 7\% annual increase in SRCs from 1988 through 2004, resulting in a $105 \%$ increase in concussions over this 15 -year period (Daneshvar et al., 2011). It is also important to recognize individual factors that may impact a person's neurocognitive performance as part of an accurate and thorough assessment of concussion. As such, the use of baseline data in concussion management is often beneficial as it allows for intra-individual comparisons that are thought to be more

Correspondence and reprint requests to: Garrett Thomas, Department of Psychology, The Pennsylvania State University, 372 Moore Building, University Park, PA 16802, USA. E-mail: gat84@psu.edu sensitive to recognition of injury and symptomatology. For example, the use of baseline data provides further clinical utility in that it accounts for individual factors that may influence neurocognitive performance, such as history of previous head injury, diagnosis of Attention Deficit Hyperactivity Disorder (ADHD), Learning Disability, cultural and language differences, and premorbid intelligence (Barr, 2003; Elbin et al., 2013; Guskiewicz et al., 2003; McCrory et al., 2013; Merritt et al., 2017; Moser et al., 2007). However, the use of baseline data typically does not directly account for the potential effects of affective symptomatology, particularly those that are comorbid. In other words, there are no specific guidelines outlining ways to appropriately interpret neurocognitive performance while specifically accounting for the potential impact of mood disturbances, to our knowledge. This may be due, in part, to a lack of clarity on how affective symptoms are related to neurocognitive performance. 


\section{Neuropsychological Performance and Depression}

Literature regarding neuropsychological performance and depression is plentiful, with research showing that depression is associated with impaired performance on several domains across ages, including executive functioning, information processing speed, psychomotor speed, verbal and visual memory recognition and recall, and working memory (Bailey et al., 2010; Basso et al., 2013; Mohn \& Rund, 2016). Multiple studies have also directly studied the neuropsychological effects of depression following concussion in collegiate athletes, demonstrating that depression is typically associated with worse neurocognitive performance (Bailey et al., 2010; Riegler et al., 2019b). Thus, it is evident that the relationship of depressive symptomatology with cognitive dysfunction is well established, though the available research presents mixed findings regarding which neurocognitive domains, such as processing speed (Bailey et al., 2010), executive function (Mohn \& Rund, 2016), and memory (McDermott \& Ebmeier, 2009; Mohn \& Rund, 2016), have the greatest associations. It is important to note that much of this research has focused on those with clinically diagnosed depression, rather than elevated depressive symptomatology. As such, the current study aims to further explore and replicate these findings in college athletes with depressive symptomatology.

\section{Neuropsychological Performance and Anxiety}

Literature regarding anxiety disorders and neuropsychological test performance is sparse, with the available research presenting mixed results. Some findings suggest that anxiety is correlated with impaired performance on memory tasks, especially pertaining to the recollection of emotionally threatening stimuli, executive functioning tasks, attention, processing speed, and inhibition/switching tasks (Clarke \& Macleod, 2013; Dorenkamp \& Vik, 2018; Langarita-Llorente \& Gracia-Garcia, 2019). However, research within this domain is complicated by the use of differing classifications, as many studies use elevated symptomatology, while others use clinical diagnoses to define 'anxiety'. Further, among those that rely upon clinical diagnoses, there are often differing results depending on the specific anxiety disorder (Airaksinen et al., 2005). Many of these studies also present conflicting results, with some suggesting that anxiety either has no effect or may even improve performance on certain neuropsychological tests (Clarke \& Macleod, 2013; Dorenkamp \& Vik, 2018; Dotson et al., 2014). For example, some authors suggest that moderate levels of anxiety improve performance on easy tasks and may improve performance on attention and processing speed tasks due to anxiety's tendency to increase effort while suppressing the processing of task-irrelevant information (Clarke \& Macleod, 2013). Some findings also suggest that moderate levels of anxiety may improve memory performance on tasks involving emotionally- or anxiety-evocative stimuli given anxious individuals' bias toward negative emotional processing (Clarke \& Macleod, 2013). Thus, given these mixed findings, it is evident that there is not a shared understanding of how, or the extent to which, anxiety symptomatology impacts neurocognitive functioning, Therefore, it is important to further assess associations of anxiety with neuropsychological performance, especially within a college athlete population.

\section{Neuropsychological Performance and Comorbid Mood Symptoms}

Considering research on anxiety and neuropsychological performance is sparse, it is not surprising that studies evaluating associations of comorbid anxiety and depression with neurocognitive functioning are also limited. A 2002 study assessing the neuropsychological test performance of depression, anxiety, and comorbid anxiety/depression in a general sample found that individuals presenting with comorbid anxiety/ depression showed an impaired ability to retrieve newly learned information in addition to impaired immediate recall and acquisition, though this was also seen in the depression group (Kizilbash et al., 2002). Another study found that those with only depression and individuals with comorbid depression/anxiety showed worse memory function than healthy controls (HCs). However, only participants with comorbid depression/anxiety demonstrated executive function impairment and psychomotor slowing. Additionally, the comorbid depression/anxiety group had more impaired scores than either the depression group or the control group (Basso et al., 2013). Based on these limited findings, it is evident that the presentation of comorbid anxiety/depression elicits a more complicated neuropsychological profile than either depression or anxiety alone. Thus, additional research regarding comorbidity is warranted to better understand how this presentation may impact athletes and their neurocognitive performance. Furthermore, in addition to the negative outcomes associated with depression alone and anxiety alone, individuals with comorbid anxiety/depression are also susceptible to an elevated risk of mortality, higher risk of suicide, higher risk of substance use/abuse, greater utilization of healthcare resources, more severe symptoms/complaints, greater overall impairment, and poorer overall outcomes (LeMoult, Castonguay, Joormann, \& McAleavey, 2013; Dahm, Wong, \& Ponsford, 2013; Pratt, Druss, Manderscheid, \& Walker, 2016) Therefore, it is imperative that those at risk for comorbidity be identified and provided proper treatment.

\section{Current Study}

To summarize, the current study aims to fill gaps in the neuropsychology and SRC literature regarding affective symptomatology, particularly comorbidity, and its association with baseline neurocognitive testing in college athletes. While the motivation for this study and potential implication are widespread and extend beyond college athletes, this population offers a unique opportunity in that they routinely 
undergo baseline neuropsychological testing as part of their continued care. Thus, this population offers access to baseline testing that may otherwise be unfeasible in a general population. The relationship between affective symptomatology and neurocognitive performance is important because if athletes experience mood symptoms at baseline, this may skew their performance and complicate interpretation of future/postconcussion testing. Further, considering the negative health outcomes associated with affective disorders, particularly those that are comorbid, it is important to recognize individuals at risk and provide proper treatment. To that end, the aims of the current study are to: 1) assess prevalence of depression, anxiety, and comorbid depression/anxiety in a baseline collegiate athlete sample and 2) examine neuropsychological performance associated with depression, anxiety, and comorbid depression/anxiety in this sample. Regarding aim 2, we hypothesize the following: a) individuals with only depression or only anxiety will perform significantly worse (i.e., obtain lower scores) on neuropsychological tests, indicating greater impairment, than individuals without these psychological symptoms; and b) individuals with comorbid anxiety/depression will perform significantly worse on neuropsychological tests compared to individuals with no psychological symptoms, only depression, or only anxiety at baseline.

\section{METHODS}

\section{Participants}

This archival study included $831(M=620, F=211)$ college athletes who were involved in a concussion management program at our Division I University. The mean age of participants was 18.50 years $(S D=1.04)$ with a range from 17 to 24. All participants were referred for baseline testing by their athletic trainer or team physician as part of standard operating procedures within the athletic programs. This neuropsychological testing included a hybrid neuropsychological test battery, psychosocial questionnaires, and information about relevant concussion history. Athletes from the following sports underwent baseline testing: football, men's and women's soccer, wrestling, men's and women's lacrosse, men's and women's ice hockey, men's and women's basketball, baseball, softball, crew, volleyball, and rugby. Athletes in the current study were selected from a larger group of athletes $(N=1050)$ receiving baseline testing between 2002 and 2019. Athletes were only included in the current study if they completed neuropsychological testing, the Beck Depression Inventory-Fast Screen (BDI-FS; Beck, Steer, \& Brown, 2000) and the NEO-Five Factor Inventory (NEO-FFI; McCrae \& Costa, 2004) at baseline. All data were collected prior to the COVID-19 pandemic.

\section{Procedures}

Baseline testing was completed as part of the SportsConcussion Program at this NCAA Division I University.
All participants completed a $2.5-\mathrm{hr}$ comprehensive neuropsychological test battery at baseline. The neuropsychological test battery was administered by undergraduate research assistants or graduate students who were supervised by a Ph.D.-level clinical neuropsychologist.

The hybrid neuropsychological battery consisted of both paper-and-pencil and computerized neuropsychological and neurobehavioral measures. The paper-and-pencil neuropsychological tests were: the Brief-Visuospatial Memory Test-Revised (BVMT-R; Benedict, 1997), the Comprehensive Trail-Making Test (CTMT; Reynolds, 2002), a modified version of the Digit Span Test (Weschler, 1997), the Hopkins Verbal Learning TestRevised (HVLT-R; Brandt \& Benedict, 2001), the Penn State University Cancellation Test (Echemendia, Putukian, Mackin, Julian, \& Shoss, 2001), the Stroop Color-Word Test (Trenerry, Crosson, DeBoe, \& Leber, 1989), and the Symbol-Digit Modalities Test (SDMT; Smith, 1991). The computerized tests were the ImPACT (Lovell, Collins, Podell, Powell, \& Maroon, 2000) and the Vigil/W Continuous Performance Test (Cegalis \& Cegalis, 1994).

The BDI-FS was used to measure depressive symptoms in this study. The BDI-FS consists of seven items rated from 0 to 3 , with higher scores indicating more severe symptoms. The BDI-FS is well validated for use in medical populations and may be a particularly useful tool for measuring depressive symptoms in concussed populations because of its ability to discriminate between depression symptoms and symptoms of a concussion (Riegler et al., 2019a).

A modified subscale of the NEO-FFI was used to measure anxiety in order to preserve sample size. The decision to utilize a subscale of the NEO-FFI was predicated upon research suggesting that neuroticism, as described in the NEO-FFI, is clinically associated with anxiety - both as a clinical disorder and as elevated symptomatology (Widiger, 2011; Kotov, Gamez, Schmidt, \& Watson, 2010; Paulus, Vanwoerden, Norton, \& Sharp, 2016). One study examining the structural relations between NEO-FFI facets and clinical diagnoses via latent regression found that neuroticism had a significant positive association with Generalized Anxiety Disorder (GAD) $(\gamma=.64)$, Social Phobia $(\gamma=.35)$, and ObsessiveCompulsive Disorder $(\gamma=.22)$ in a clinical sample (Rosellini \& Brown, 2011). Moreover, higher levels of anxiety and neuroticism have been linked to risk of injury and reinjury in athletes (Brewer, 2007; Ford et al., 2017). Additionally, studies have found that anxiety may also impede recovery from injuries, including both orthopedic injuries and concussion (Covassin et al., 2014). Thus, the relationship between anxiety and injury risk is worth exploring, especially considering that early identification of athletes who are potentially at increased risk for subsequent injurious events may benefit from early intervention - either pre- or post-injury. Therefore, further exploring the impacts of neuroticism and anxiety is especially pertinent for the current study. The NEO-FFI Anxiety Subscale was created using the four anxiety items found in the neuroticism facet of the NEO-FFI. This subscale was created in order to obtain a purer 
anxiety measure, as the neuroticism facet encompasses both depression and anxiety. The four items included in the anxiety subscale were as follows: "I am not a worrier", "When I'm under a great deal of stress, sometimes I feel like I'm going to pieces," "I often feel tense and jittery," and "I rarely feel fearful or anxious." In order to validate the content of this subscale, we compared it to the Anxiety Sensitivity Index (ASI: Reiss, Peterson, Gursky, \& McNally, 1986) in a separate database containing a similar college-age population which can be considered a reasonably equivalent sample. In this sample, the NEO-FFI Anxiety Subscale was significantly correlated with the ASI $(r=.35, p=.014)$, which is similar to the ASI's correlation with other trait anxiety measures in previous research $(r=.42, r=.43, r=.46)$ (McNally $\&$ Lorenz, 1987; Reiss et al., 1986; Sandin et al., 2001). The ASI has demonstrated sensitivity in distinguishing between anxiety disorders like GAD, Social Phobia, and Panic Disorder (Rodriguez et al., 2004). The ASI has also shown reliability in assessing anxiety in college students (Peterson $\&$ Heilbronner, 1987). Thus, the ASI appears to be an appropriate comparison for validation, and the NEO-FFI Anxiety Subscale appears to be an acceptable measure of anxiety for the current study.

Considering that there is not a validated cut-off for the NEO-FFI Anxiety Subscale, the current study used a score of one standard deviation above the sample mean $(M=6.58, S D=2.78)$ to indicate clinical significance. As such, NEO-FFI Anxiety Subscale scores were dichotomized into two groups based on the presence of clinically meaningful anxiety symptomatology $(\geq 10)$ or absence of clinically meaningful anxiety symptomatology $(<10)$. The decision to use one standard deviation above the mean as a cut-off score is predicated upon our interest in being able to include those who report mild-moderate symptoms of anxiety, in addition to those with more severe symptoms. This is due, in part, to our interest in exploring the impact of elevated affective symptomatology rather than only including those who meet clinical diagnostic criteria.

While the distribution of NEO-FFI Anxiety Subscale scores was normally distributed, the distribution of the BDI-FS scores was not normal and showed positive skewness and kurtosis in this sample. Further, a large percentage of individuals scored 0 on the BDI-FS. Given these considerations, and in order to maintain consistency with the cut-offs for the NEO-FFI subscale, the BDI-FS cut-offs were derived based on scores one standard deviation greater than the sample mean $(M=1.08, S D=1.77)$. As such, BDI-FS scores were dichotomized into two groups based on the presence of clinically meaningful depressive symptomatology $(\geq 3)$ or absence of clinically meaningful depressive symptomatology $(<3)$. In addition to the considerations noted above, the decision to dichotomize depression and anxiety in this way was predicated upon our specific interest in evaluating differences between groups, particularly the comorbid depression/anxiety group compared to the other groups. The control, depression only, anxiety only, and comorbid depression and anxiety groups are defined in Table 1 . The
Table 1. Definition of experimental groups determined by clinical measures

\begin{tabular}{lcc}
\hline \hline & BDI-FS & NEO-FFI Anxiety Subscale \\
\hline Healthy control & $<3$ & $<10$ \\
Depression only & $\geq 3$ & $<10$ \\
Anxiety only & $<3$ & $\geq 10$ \\
Comorbid depression & $\geq 3$ & $\geq 10$ \\
$\quad$ and anxiety & & \\
\hline \hline
\end{tabular}

groups did not differ in terms of mean age, mean estimated FSIQ, or proportion of athletes who reported previous concussion(s).

All participants provided written informed consent, and the study was approved by the Behavioral Committee of the Institutional Review Board at our university.

\section{Calculation of $Z$ scores}

Scores on all neuropsychological test indices were standardized to $Z$ scores using published baseline norms from a large sample of college athletes from a Division I university (Merritt et al., 2017; Riegler et al., 2019b). Merritt et al. (2017) provided normative data for males $(N=577)$ and females $(N=217)$ separately on all measures of interest except for the ImPACT test, while Riegler et al. (2019b) provided normative data for males $(N=893)$ and females $(N=377)$ on the ImPACT test.

\section{Calculation of composite scores}

Principal Component Analyses (PCAs) were conducted to identify and create composite scores for conceptually related test indices (attention/processing speed tests and memory tests). The PCA for the attention/processing speed composite included the following indices: ImPACT Reaction Time Composite, ImPACT Visual Motor Speed Composite, Vigil Omissions, Vigil Commissions, Vigil Average Delay, SDMT Total, Stroop 1 and 2 Time, PSU Cancellation, CTMT "Simple," CTMT "Executive," Digits Forward, and Digits Backward. Of the 13 tests entered into the analysis, 10 of the variables loaded above .40 and were retained for the final Attention/Processing Speed Composite. The indices eliminated included: ImPACT Reaction Time Composite, Vigil Omissions, and Vigil Commissions. A comparable PCA was conducted with the following memory indices: ImPACT Verbal Memory Composite, ImPACT Visual Memory Composite, BVMT-R Total Immediate and Delayed Recall, and HVLT-R Total Immediate and Delayed Recall. All variables loaded above .55 and were retained for the final Memory Composite.

Following the PCA, the final composites were calculated by first standardizing the individual indices comprising the composites to $Z$ scores and then calculating a mean $Z$ score 
Table 2. Prevalence rates of affective symptoms

\begin{tabular}{lcrrrr}
\hline \hline & & \multicolumn{5}{c}{ Sex } \\
\cline { 3 - 6 } & \begin{tabular}{c} 
Mean \\
age \\
\cline { 3 - 6 }
\end{tabular} & & & & $\begin{array}{c}\text { \% of sample } \\
\text { population }\end{array}$ \\
\hline Baseline & Male & Female & $N^{1}$ & populary \\
Healthy control & 18.52 & 452 & 126 & 578 & 69.5 \\
Depression only & 18.57 & 103 & 34 & 137 & 16.5 \\
Anxiety only & 18.28 & 29 & 25 & 54 & 6.5 \\
Comorbid depres- & 18.44 & 36 & 26 & 62 & 7.5 \\
$\quad$ sion and anxiety & & & & & \\
\hline \hline
\end{tabular}

${ }^{1}$ These groups are all independent, meaning that athletes captured in the depression only or anxiety only groups are not counted toward the comorbid group. In doing so, we attempted to make the groups as diagnostically pure as possible. For example, the $n$ of the anxiety only group is smaller than the comorbid group because many of the athletes experiencing symptoms of anxiety also experience symptoms of depression, thus falling into the comorbid group and not the anxiety only group.

value for each composite. Thus, we ended up with an Attention/Processing Speed composite (10 indices) and Memory composite (6 indices).

\section{RESULTS}

\section{Prevalence Rates}

Approximately $69.5 \%$ of our sample population did not experience significant affective symptomatology, whereas $30.5 \%$ of athletes met criteria for at least one significant affective disturbance at baseline. Specifically, $16.5 \%$ of the population experienced depressive symptoms without anxiety symptoms, $6.5 \%$ of the population experienced anxiety symptoms without depressive symptoms, and $7.5 \%$ of the population experienced both depressive and anxiety symptoms (Table 2).

\section{Neurocognitive Performance}

To examine the impact of affective symptomatology, we first conducted a regression with a priori planned orthogonal contrast coding to examine differences in baseline performance between groups on the Attention/Processing Speed and Memory Composites. The decision to use orthogonal contrast coding in the first regression, as opposed to an analysis of variance and post hoc test approach, was in order to maximize statistical power and allow for meaningful combining of the groups (e.g., combining the three affective groups to compare to $\mathrm{HCs}$ ).

Overall, results revealed a marginally significant effect of Group (as determined by affective symptomatology) on Attention/Processing Speed, $F(3,827)=2.58, p=.053$, $\eta^{2}=.01$, but not on Memory, $F(3,827)=.69, p=.56$, $\eta^{2}=.003$. We found that the Attention/Processing Speed Composite was significantly lower across the combined means of the affective groups $(M=-.10)$ compared with the HC group $(M=.01), t(827)=2.66, p=.01, d=.19$.
However, there was no significant difference between affective groups $(M=-.03)$ and the HC group $(M=.01)$ on the Memory Composite, $t(827)=1.06, p=.29, d=.06$. We also found that while the comorbid group tended to score lower on measures of Attention/Processing Speed and Memory compared to the depressive symptoms and anxiety symptoms groups, the mean differences were not statistically significant. Further, the depressive symptoms only group and anxiety symptoms only group performed similarly across both indices.

To further examine differences between groups, we replicated the previous regression using dummy coding instead of complex contrast coding, which also allowed us to maximize statistical power while allowing for meaningful comparisons. For example, this approach allowed us to directly compare each of the affective groups to the HC group, rather than evaluating the combined group means. These comparisons were planned a priori.

\section{Comorbid group versus HC group}

The Attention/Processing Speed Composite was significantly lower in the comorbid group $(M=-.16)$ compared with the HC group $(M=.01), t(827)=-2.28, p=.02, d=.29$. There was no significant difference between the comorbid group and the $\mathrm{HC}$ group on the Memory Composite, $t(827)=-1.43, p=.15, d=.19$.

\section{Depressive symptoms only group versus HC group}

While the depressive symptoms only group scored worse than the HC group on measures of Attention/Processing Speed and Memory, the mean differences were not significantly different for either of the composite scores (Table 3).

\section{Anxiety symptoms only group versus $H C$ group}

Similar to the depressive symptoms only group, while the anxiety symptoms only group scored worse than the HC group on measures of Attention/Processing Speed and Memory, the mean differences were not significantly different for either of the composite scores (Table 3).

In sum, while the combined mean performance of all affective groups was significantly lower than the HC group on measures of Attention/Processing Speed at baseline, only the comorbid group was significantly different from the HC group when compared individually. However, the comorbid group was not significantly different from the other affective groups.

\section{Proportion of Neurocognitively Impaired versus Not Impaired Athletes}

We also conducted Chi-square analyses to explore the proportion of neurocognitively impaired athletes within each group determined by two separate criteria. 
Table 3. Group performance on cognitive indices ( $Z$ scores)

\begin{tabular}{|c|c|c|c|c|c|c|}
\hline Composite & $n$ & Mean & $S D$ & $t^{a}$ & $p$-value ${ }^{a}$ & $(d)^{\mathrm{a}, \mathrm{b}}$ \\
\hline \multicolumn{7}{|c|}{ Attention/Processing Speed } \\
\hline $\begin{array}{l}\text { Healthy control } \\
\text { (HC) }\end{array}$ & 578 & .01 & .55 & - & - & - \\
\hline Depression & 137 & -.07 & .61 & -1.59 & .11 & .14 \\
\hline Anxiety & 54 & -.09 & .65 & -1.28 & .20 & .17 \\
\hline Comorbid & 62 & -.16 & .63 & -2.28 & $.02^{*}$ & .29 \\
\hline Combined groups ${ }^{c}$ & 253 & -.10 & .62 & 2.66 & $.01^{*}$ & .19 \\
\hline \multicolumn{7}{|l|}{ Memory } \\
\hline $\begin{array}{l}\text { Healthy control } \\
\text { (HC) }\end{array}$ & 578 & .01 & .67 & - & - & - \\
\hline Depression & 137 & .001 & .65 & -.17 & .87 & .01 \\
\hline Anxiety & 54 & -.02 & .62 & -.34 & .74 & .05 \\
\hline Comorbid & 62 & -.12 & .69 & -1.43 & .15 & .19 \\
\hline Combined groups $^{c}$ & 253 & -.03 & .66 & 1.06 & .29 & .06 \\
\hline
\end{tabular}

${ }^{\mathrm{a}}$ Compared to healthy control group.

${ }^{\mathrm{b}}$ Cohen's effect sizes: small (.2), medium (.5), and large (.8) (Cohen, 2013).

${ }^{\mathrm{c}}$ Combined groups include the depression only, anxiety only, and comorbid groups.

*Significant at the .05 level.

\section{Global impairment based on algorithm}

Athletes were considered neurocognitively impaired based on criteria outlined in Arnett et al. (2016). Based on this algorithm involving 17 test indices ${ }^{1}$, athletes were considered "neurocognitively impaired" if they scored 1.5 standard deviations or more below the mean on $5+$ indices for males and $3+$ indices for females, or if they scored 2 standard deviations or more below the mean on $3+$ indices for males and $2+$ indices for females (Arnett et al., 2016). Athletes who were classified as impaired by both criteria were only counted once. We found that, compared to the HC group, the comorbid group had significantly more athletes classified as neurocognitively impaired, $\chi^{2}(1, N=640)=10.12, p=.001, \phi=.13^{2}$, as did the depressive symptoms only group, $\chi^{2}(1$, $N=715)=4.61, p=.03, \phi=.08$, and the anxiety symptoms only group, $\chi^{2}(1, N=632) 5.57, p=.02, \phi=.09$. See Figure 1 and Table 4.

\section{Impairments based on algorithm applied to separate composites}

To further explore these findings regarding algorithmderived impairments based on all 17 test indices, we created new decision rules for the Attention/Processing Speed Composite and Memory Composites separately. These new decision rules emulated the original algorithm described in Arnett et al. (2016) in that "impaired" scores were defined

\footnotetext{
${ }^{1}$ The indices included in this algorithm are the same as the indices included in the previous analyses for this paper; however, the algorithm also includes the ImPACT Reaction Time composite. This measure was not included in our initial analyses because it loaded below the 0.4 cutoff for the PCA, but we included it here to be more analogous with the published algorithm.

${ }^{2}$ Phi effect size for Chi-Square: small (0.1), medium (0.3), large (0.5) (Cohen, 2013; Kim, 2017)
}

as performing $2 \mathrm{SDs}$ or more below the sample mean, and "borderline" scores were defined as performing 1.5 SDs or more below the sample mean. Contrary to the original algorithm, there were no sex differences in terms of base rate of impairment, so males and females were included within the same decision rules. The overall sample used to determine these cut-offs included 919 college athletes $(M=685$, $F=234$ ). Overall, fewer than $10 \%$ of athletes had 3 or more borderline scores on the Attention/Processing Speed Composite, and fewer than $10 \%$ of athletes had 3 or more borderline scores on the Memory Composite. Additionally, fewer than $10 \%$ of athletes had 2 or more impaired scores on the Attention/Processing Speed Composite, and fewer than $10 \%$ of athletes had 2 or more impaired scores on the Memory Composite. As such, these cut-offs were used to define athletes as either Neurocognitively Impaired or Not Impaired. The Neurocognitively Impaired Group were those who either showed $3+$ borderline indices or $2+$ impaired indices. Athletes meeting both criteria were only counted once. Conversely, those in the Not Impaired Group were athletes who showed $<3$ borderline indices and $<2$ impaired indices.

For Chi-square analyses, athletes were separated into two groups based on the criteria described above. Dichotomizing groups and conducting Chi-square analyses in this way have been utilized in several previously published studies regarding neurocognitive performance (Guty \& Arnett, 2018; Merritt et al., 2018; Rabinowitz \& Arnett, 2013; Riegler et al., 2019b). Additionally, this approach has shown clinical utility in that it allows for a more nuanced understanding of an athlete's individual presentation compared to the sole reliance upon mean performance scores, which can sometimes obscure important clinical findings. We found that, compared to the HC group, the comorbid group had significantly more athletes classified as neurocognitively impaired on the Attention/Processing Speed composite, $\chi^{2}(1, N=640)=$ $11.81, p=.001, \phi=.14$, but not on the Memory composite, $\chi^{2}(1, N=640)=.64, p=.42, \phi=.03$. Similarly, the anxiety symptoms only group had significantly more athletes classified as neurocognitively impaired than the $\mathrm{HC}$ group on the Attention/Processing Speed composite, $\chi^{2}(1, N=632)=$ $4.98, p=.03, \phi=.09$, but not on the Memory composite, $\chi^{2}(1, N=632)=.15, p=.70, \phi=.02$. The proportion of neurocognitively impaired athletes in the depressive symptoms only group did not differ from that of the HC group on either of the composites. See Figure 2 and Table 4.

\section{DISCUSSION}

Overall, these findings indicate that approximately $30 \%$ of the sample population reported experiencing at least one significant affective disturbance (e.g. depressive, anxious, or comorbid symptomatology) at baseline. This percentage is slightly lower, but consistent, with recent research surrounding the prevalence of affective disorders in college students (Beiter et al., 2015; Duffy et al., 2019). Additionally, the results indicate that comorbid depression and anxiety are 
Table 4. Proportion of neurocognitively impaired athletes per group

\begin{tabular}{|c|c|c|c|c|c|}
\hline Group & Impaired & Not impaired & $\chi^{2}$ & $p$ & $\phi^{\mathrm{c}}$ \\
\hline \multicolumn{6}{|l|}{ Algorithm derived $^{a}$} \\
\hline Healthy control (HC) & $43(7.4 \%)$ & 535 & - & - & \\
\hline Depression vs. HC & $18(13.1 \%)$ & 119 & 4.61 & $.03^{*}$ & .08 \\
\hline Anxiety vs. HC & $9(16.7 \%)$ & 45 & 5.57 & $.02^{*}$ & .09 \\
\hline Comorbid vs. HC & $12(19.4 \%)$ & 50 & 10.12 & $.001^{*}$ & .13 \\
\hline \multicolumn{6}{|c|}{ Algorithm derived by composite ${ }^{b}$} \\
\hline \multicolumn{6}{|c|}{ Attention/Processing Speed } \\
\hline Healthy control (HC) & $45(7.8 \%)$ & 533 & - & - & \\
\hline Depression vs. HC & $13(9.5 \%)$ & 124 & .43 & .51 & .03 \\
\hline Anxiety vs. HC & $9(16.7 \%)$ & 45 & 4.98 & $.03^{*}$ & .09 \\
\hline Comorbid vs. HC & $13(21.0 \%)$ & 49 & 11.81 & $.001^{*}$ & .14 \\
\hline \multicolumn{6}{|l|}{ Memory } \\
\hline Healthy control (HC) & $42(6.9 \%)$ & 536 & - & - & \\
\hline Depression vs. HC & $11(7.3 \%)$ & 126 & .02 & .88 & .01 \\
\hline Anxiety vs. HC & $4(5.6 \%)$ & 50 & .15 & .70 & .02 \\
\hline Comorbid vs. HC & $6(9.7 \%)$ & 56 & .64 & .42 & .03 \\
\hline
\end{tabular}

a Athletes were considered "neurocognitively impaired" if they scored 1.5 standard deviations or more below the mean on $5+$ indices for males and $3+$ indices for females or if they scored 2 standard deviations or more below the mean on $3+$ indices for males and $2+$ indices for females.

b Athletes were considered "neurocognitively impaired" if they scored 1.5 standard deviations or more below the mean on $3+$ indices or if they scored 2 standard deviations or more below the mean on $2+$ for each of the composites.

${ }^{\mathrm{c}}$ Phi effect size for Chi-square: small (.1), medium (.3), and large (.5) (Cohen, 2013; Kim, 2017).

*Significant at the .05 level.

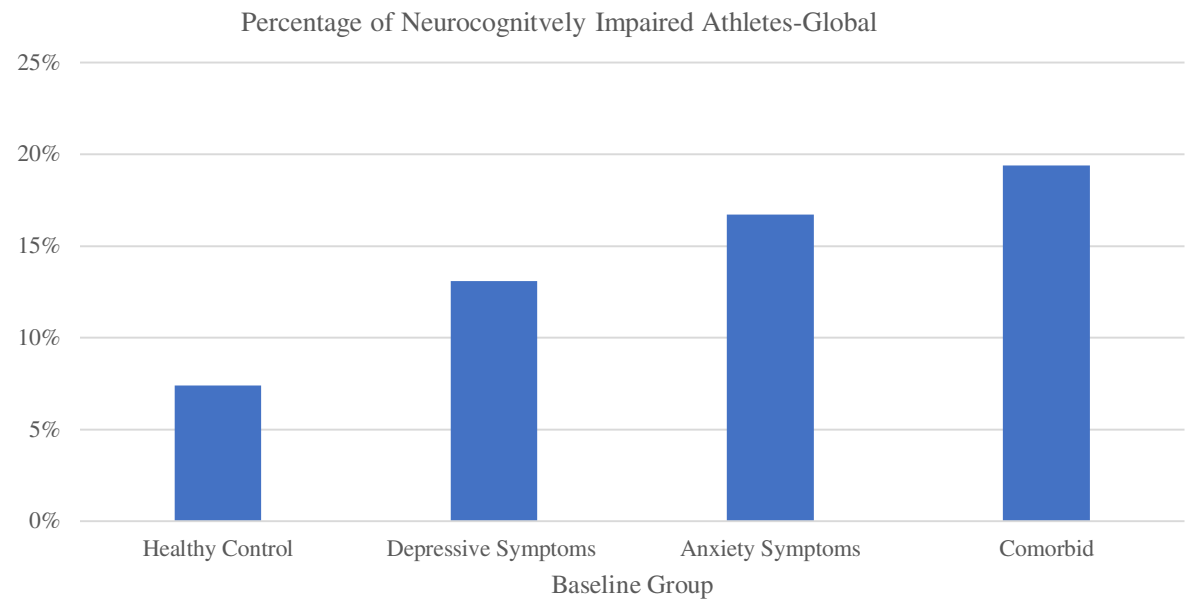

Fig. 1. Percentage of neurocognitively impaired athletes at baseline as determined by the global algorithm. The specific percentages are found in Table 4.

associated with poorer neurocognitive performance in areas of Attention/Processing Speed. Our first hypothesis was partially supported, as both the depressive symptoms only and anxiety symptoms only groups had a significantly greater proportion of neurocognitively impaired athletes compared with the HC group when applying the global impairment algorithm. Moreover, when classifying impairment based on the algorithm for the Attention/Processing Speed and Memory composites separately, the anxiety symptoms only group showed a significantly greater proportion of athletes who were impaired on Attention/Processing Speed compared to HCs. Of note, the anxiety symptoms only group also showed a higher proportion of athletes who reported a previous ADHD diagnosis compared to other groups, though the number of athletes with ADHD who were also classified as impaired did not significantly differ between groups. Thus, it does not appear that a previous diagnosis of ADHD is sufficient to explain these findings. Moreover, despite these findings, the depressive symptoms only and anxiety symptoms only groups did not show mean values that were significantly lower than HCs on measures of Attention/Processing Speed or Memory. This suggests that athletes within these groups performed poorly on several indices, thus leading to "impaired" classification based on number of indices with impaired scores, but they perhaps "made up" for this on other indices in the battery, thus leading to a mean score that was 


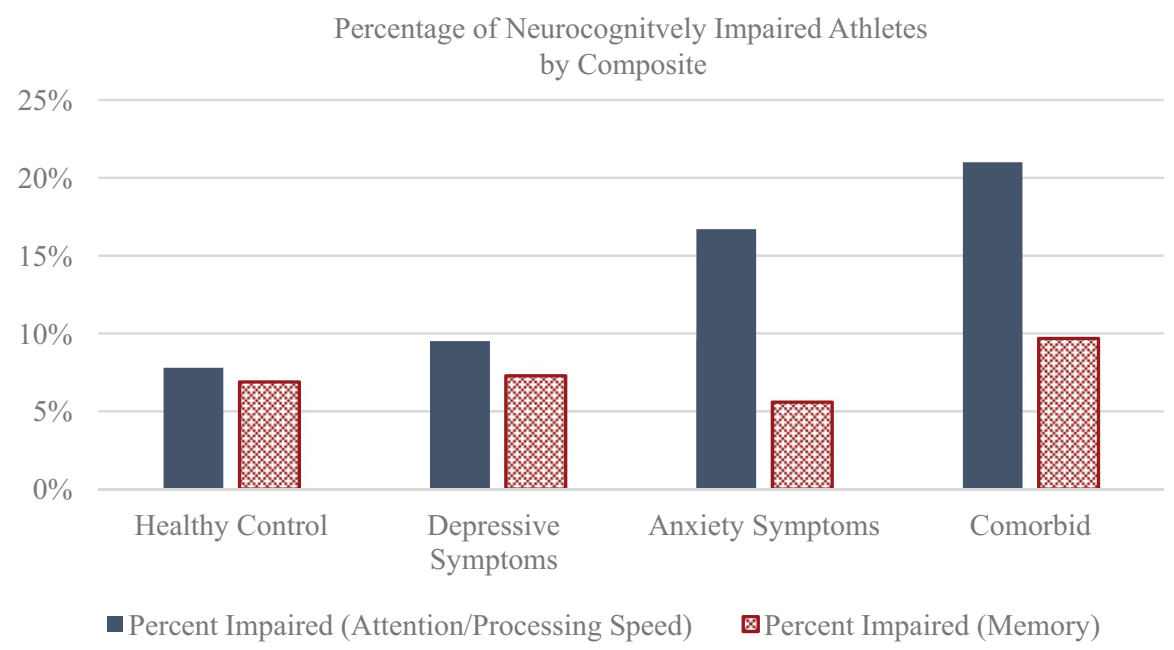

Fig. 2. Percentage of neurocognitively impaired athletes at baseline as determined by the separate composite algorithms. Specific percentages are found in Table 4.

within the range of HCs. This potential explanation offers further support for utilizing an algorithm-based approach in that it allows for an understanding of athletes' presentations at a more nuanced and individualized level and can help identify those who may otherwise be missed when solely relying upon mean scores.

Our second hypothesis was also partially supported in that the Comorbid Depression/Anxiety group performed significantly worse than HCs on measures of Attention/ Processing Speed. The comorbid group also had nearly three times as many neurocognitively impaired athletes compared to the $\mathrm{HC}$ group when applying the algorithm for global impairment. Additionally, compared with the HC group, the comorbid group had nearly three times as many athletes who were impaired on the Attention/Processing Speed composite. Interestingly, the comorbid group did not perform significantly worse than the HCs on measures of Memory, nor did the comorbid group differ from the HC group in the proportion of athletes classified as impaired on the Memory composite. These findings were unexpected given previous research regarding affective symptomatology and its association with memory functioning (Riegler et al., 2019b). This may be due, at least in part, to using a more moderate cut-off for classifying depression compared to other studies. Further research regarding comorbidity and its effects on memory, in addition to research regarding the impact of moderate depression as opposed to clinical depression, is warranted. Additionally, the comorbid group did not perform significantly worse than the depressive symptoms only and anxiety symptoms only groups on measures of Attention/Processing Speed or Memory. This could be due, in part, to the separate effects of depressive symptoms and anxiety symptoms being relatively similar, but not as potent, as the cumulative effects of comorbidity as compared to HCs. In other words, while depression or anxiety alone did not elicit neurocognitive differences compared to $\mathrm{HCs}$, comorbidity did lead to a significant difference compared to HCs. Thus, there appears to be an additive effect in that experiencing both depression and anxiety elicits a significant effect that is not seen with either depression or anxiety alone when compared to HCs.

One possible limitation of the current study is the use of the anxiety subscale, which was derived from the neuroticism facet of the NEO-FFI, rather than a stand-alone anxiety measure. While this anxiety subscale did correlate with the ASI at a level comparable to other anxiety measures (e.g. the STAI), the scale we derived has not been subject to rigorous validation and may be a limitation. Another related limitation lies within the clinical utility of these findings. Because we created the affective groups based on cut-off scores derived from the sample mean and standard deviation, rather than clinical cut-offs, we captured those who were experiencing affective symptomatology rather than true clinical disorders. Still, we believe that these cut-offs are clinically meaningful because they are derived from base rates within our sample, and also because of some athletes' tendency to underreport symptoms (McCrea et al., 2004; Meier et al., 2015). Future studies could use more robust cut-offs, especially in regard to anxiety symptomatology, to more fully assess the impact of affective symptoms and disorders on neurocognitive performance. This study is also limited in that we are not able to fully account for potential external influences, specifically pharmacological substances, on neurocognitive performance. Thus, there may be factors other than depression and anxiety that contribute to these findings.

In sum, our results show that significant affective symptomatology is common in collegiate athletes at baseline. Our data also highlight the negative impact of affective symptomatology, particularly comorbidity, on neurocognitive performance at baseline in collegiate athletes. As such, baseline testing might be more critical for athletes who have mood symptoms in order to capture a more complete understanding 
of their cognitive profile. For example, if an athlete meets criteria for psychiatric comorbidity at baseline, but not post-concussion, this could give a skewed representation of their functioning and may result in returning athletes prior to cognitive recovery. With this in mind, neuropsychologists are in a unique position to capture a truly individualistic understanding of an athlete's presentation through baseline testing and psychological interviewing. The sole reliance upon selfreport to identify psychological distress may not be sufficient considering some athletes may underreport symptoms, including psychological symptoms, due to motivation to return to play (Echemendia \& Cantu, 2003; McCrea, Hammeke, Olsen, Leo, \& Guskiewicz, 2004; Meier et al., 2015; Riegler, Guty, \& Arnett, 2019b). One study found that athletes reported significantly more psychological symptoms, including symptoms of anxiety and depression, in a confidential psychological interview than they reported to athletic trainers via self-report on the ImPACT (Meier et al., 2015). As such, introducing a brief screener, like the NEO-FFI Anxiety Subscale, BDI-FS, and/or the Affective Scale from the PCSS (Riegler et al., 2019a), may be a good way to identify athletes who might be at risk for affective symptomatology who can then be referred for a more thorough assessment.

Given these considerations, athletes should be routinely screened for mood disorders and those who show affective symptomatology could receive baseline testing even if it is not standard procedure. This is important because post-concussion assessments are more meaningful if they can be compared to a valid baseline. If an individual's possible psychopathology is not accounted for, these affective symptoms may skew baseline performance, so that comparison with assessments at future time points may not be accurate.

\section{ACKNOWLEDGEMENTS}

The authors do not have a financial investment in this study or the outcome of the data to report.

\section{CONFLICT OF INTEREST}

None.

\section{REFERENCES}

Airaksinen, E., Larsson, M., \& Forsell, Y. (2005). Neuropsychological functions in anxiety disorders in population-based samples: Evidence of episodic memory dysfunction. Journal of Psychiatric Research, 39(2), 207-214. doi: 10.1016/ j.jpsychires.2004.06.001.

Arnett, P., Meyer, J., Merritt, V., \& Guty, E. (2016). Neuropsychological testing in mild traumatic brain injury: what to do when baseline testing is not available. Sports Medicine and Arthroscopy Review, 24(3), 116-122. doi: 10.1097/JSA. 0000000000000123.

Bailey, C.M., Samples, H.L., Broshek, D.K., Freeman, J.R., \& Barth, J.T. (2010). The relationship between psychological distress and baseline sports-related concussion testing. Clinical
Journal of Sport Medicine: Official Journal of the Canadian Academy of Sport Medicine, 20(4), 272-277. doi: 10.1097/ JSM.0b013e3181e8f8d8.

Barr, W.B. (2003). Neuropsychological testing of high school athletes Preliminary norms and test-retest indices. Archives of Clinical Neuropsychology, 18(1), 91-101. doi: 10.1093/arclin/ 18.1.91.

Basso, M.R., Miller, A., Estevis, E., \& Combs, D. (2013). Neuropsychological deficits in major depressive disorder: correlates and conundrums. In P. A. Arnett (Ed.), Secondary Influences on Neuropsychological Test Performance (pp. 39-66). New York, NY: Oxford University Press.

Beck, A., Steer, R., \& Brown, G. (2000). BDI-Fast Screen for Medical Patients Manual. San Antonio, TX: Psychological Corporation.

Beiter, R., Nash, R., McCrady, M., Rhoades, D., Linscomb, M., Clarahan, M., \& Sammut, S. (2015). The prevalence and correlates of depression, anxiety, and stress in a sample of college students. Journal of Affective Disorders, 173, 90-96. doi: 10.1016/j. jad.2014.10.054.

Benedict, R.H.B. (1997). Brief Visuospatial Memory Test-Revised: Professional Manual. Odessa, FL: Psychological Assessment Resource.

Brandt, J., \& Benedict, R.H.B. (2001). Hopkins Verbal Learning Test-Revised (HVLT-R). Odessa, FL: Psychological Assessment Resources.

Brewer, B.W. (2007). Psychology of sport injury rehabilitation. In G. Tenenbaum \& R. C. Eklund (Eds.), Handbook of Sport Psychology, 3rd ed., (pp. 404-424). Hoboken, NJ: John Wiley \& Sons, Inc.

Cegalis, J.A., \& Cegalis, S. (1994). The Vigil/W Continuous Performance Test (manual). New York, NY, US: For Thought.

Clarke, P. \& Macleod, C. (2013). The impact of anxiety on cognitive task performance. In P. A. Arnett (Ed.), Secondary Influences on Neuropsychological Test Performance, (pp. 93-116). New York, NY: Oxford University Press.

Cohen, J. (2013). Statistical Power Analysis for the Behavioral Sciences. Cambridge, MA: Academic Press.

Covassin, T., Crutcher, B., Bleecker, A., Heiden, E. O., Dailey, A., \& Yang, J. (2014). Postinjury anxiety and social support among collegiate athletes: a comparison between orthopaedic injuries and concussions. Journal of Athletic Training, 49(4), 462-468. doi: 10.4085/1062-6059-49.2.03.

Dahm, J., Wong, D., \& Ponsford, J. (2013). Validity of the depression anxiety stress scales in assessing depression and anxiety following traumatic brain injury. Journal of Affective Disorders, 151(1), 392-396. doi: 10.1016/j.jad.2013.06.011.

Daneshvar, D.H., Nowinski, C.J., McKee, A., \& Cantu, R.C. (2011). The epidemiology of sport-related concussion. Clinics in Sports Medicine, 30(1), 1-17. doi: 10.1016/j.csm.2010.08.006.

Dorenkamp, M.A. \& Vik, P. (2018). Neuropsychological assessment anxiety: a systematic review. Practice Innovations, 3(3), 192-211. http://dx.doi.org.ezaccess.libraries.psu.edu/10.1037/ pri0000073

Dotson, V.M., Szymkowicz, S.M., Kirton, J.W., McLaren, M.E., Green, M.L., \& Rohani, J.Y. (2014). Unique and interactive effect of anxiety and depressive symptoms on cognitive and brain function in young and older adults. Journal of Depression \& Anxiety, Suppl 1. doi: 10.4172/2167-1044.S1-003.

Duffy, M.E., Twenge, J.M., \& Joiner, T.E. (2019). Trends in Mood and anxiety symptoms and suicide-related outcomes among U.S. 
undergraduates, 2007-2018: evidence from two national surveys. Journal of Adolescent Health, 65(5), 590-598. doi: 10.1016/j. jadohealth.2019.04.033.

Echemendia, R.J. \& Cantu, R.C. (2003). Return to play following sports-related mild traumatic brain injury: the role for neuropsychology. Applied Neuropsychology, 10(1), 48-55. doi: 10.1207/ S15324826AN1001_7.

Echemendia, R.J., Putukian, M., Mackin, R.S., Julian, L., \& Shoss, N. (2001). Neuropsychological test performance prior to and following sports-related mild traumatic brain injury. Clinical Journal of Sport Medicine, 11, 23.

Elbin, R.J., Kontos, A.P., Kegel, N., Johnson, E., Burkhart, S., \& Schatz, P. (2013). Individual and combined effects of LD and ADHD on computerized neurocognitive concussion test performance: evidence for separate norms. Archives of Clinical Neuropsychology, 28(5), 476-484. doi: 10.1093/arclin/ act024.

Ford, J.L., Ildefonso, K., Jones, M.L., \& Arvinen-Barrow, M. (2017). Sport-related anxiety: current insights. Open Access Journal of Sports Medicine, 8, 205-212. doi: 10.2147/OAJSM. S125845.

Guskiewicz, K.M., McCrea, M., Marshall, S.W., Cantu, R.C., Randolph, C., Barr, W., Onate, J.A., \& Kelly, J.P. (2003). Cumulative effects associated with recurrent concussion in collegiate football players: the NCAA concussion study. JAMA, 290(19), 2549-2555. doi: 10.1001/jama.290.19.2549.

Guty, E. \& Arnett, P. (2018). Post-concussion symptom factors and neuropsychological outcomes in collegiate athletes. Journal of the International Neuropsychological Society: JINS, 24(7), 684-692. doi: 10.1017/S135561771800036X.

Kim, H.-Y. (2017). Statistical notes for clinical researchers: chi-squared test and Fisher's exact test. Restorative Dentistry \& Endodontics, 42(2), 152-155. doi: 10.5395/rde.2017.42.2.152.

Kizilbash, A.H., Vanderploeg, R.D., \& Curtiss, G. (2002). The effects of depression and anxiety on memory performance. Archives of Clinical Neuropsychology, 17(1), 57-67. doi: 10. 1093/arclin/17.1.57.

Kotov, R., Gamez, W., Schmidt, F., \& Watson, D. (2010). Linking "big" personality traits to anxiety, depressive, and substance use disorders: a meta-analysis. Psychological Bulletin, 136(5), 768-821. http://dx.doi.org.ezaccess.libraries.psu.edu/10. 1037/a0020327.

Langarita-Llorente, R. \& Gracia-Garcia, P. (2019). Neuropsychology of generalized anxiety disorders: a systematic review. Revista De Neurologia, 69(2), 59-67. doi: 10.33588/rn.6902.2018371.

Langlois, J.A., Rutland-Brown, W., \& Wald, M.M. (2006). The epidemiology and impact of traumatic brain injury: a brief overview. The Journal of Head Trauma Rehabilitation, 21(5), 375-378.

LeMoult, J., Castonguay, L. G., Joormann, J., \& McAleavey, A. (2013). Depression. In L. G. Castonguay \& T. F. Oltmanns (Eds.), Psychopathology: From science to clinical practice (pp. 17-61). New York, NY: The Guilford Press.

Lovell, M.R., Collins, M.W., Podell, K., Powell, J., \& Maroon, J.C. (2000). ImPACT: Immediate Post-Concussion assessment and cognitive testing. Pittsburgh, PA: NeroHealth Systems, LLC.

McCrae, R.R. \& Costa, P.T. (2004). A contemplated revision of the NEO five-factor inventory. Personality and Individual Differences, 36(3), 587-596. doi: 10.1016/S0191-8869(03) 00118-1.

McCrea, M., Hammeke, T., Olsen, G., Leo, P., \& Guskiewicz, K. (2004). Unreported concussion in high school football players: Implications for prevention. Clinical Journal of Sport
Medicine: Official Journal of the Canadian Academy of Sport Medicine, 14(1), 13-17.

McCrory, P., Meeuwisse, W.H., Aubry, M., Cantu, R.C., Dvorák, J., Echemendia, R.J., ... Turner, M. (2013). Consensus statement on concussion in sport-the 4th International Conference on Concussion in Sport Held in Zurich, November 2012. $P M \& R$, 5(4), 255-279. doi: 10.1016/j.pmrj.2013.02.012.

McDermott, L.M. \& Ebmeier, K.P. (2009). A meta-analysis of depression severity and cognitive function. Journal of Affective Disorders, 119(1-3), 1-8. doi: 10.1016/j.jad.2009. 04.022 .

McNally, R.J. \& Lorenz, M. (1987). Anxiety sensitivity in agoraphobics. Journal of Behavior Therapy and Experimental Psychiatry, 18(1), 3-11. doi: 10.1016/00057916(87)90065-6.

Meier, T.B., Brummel, B.J., Singh, R., Nerio, C.J., Polanski, D.W., \& Bellgowan, P.S.F. (2015). The underreporting of self-reported symptoms following sports-related concussion. Journal of Science and Medicine in Sport, 18(5), 507-511. doi: 10.1016/j. jsams.2014.07.008.

Merritt, V.C., Clark, A.L., Sorg, S.F., Evangelista, N.D., Werhane, M., Bondi, M.W., Schiehser, D.M., \& Delano-Wood, L. (2018). Apolipoprotein E $€ 4$ genotype is associated with elevated psychiatric distress in veterans with a history of mild to moderate traumatic brain injury. Journal of Neurotrauma, 35(19), 2272-2282. doi: 10.1089/neu.2017.5372.

Merritt, V.C., Meyer, J.E., Cadden, M.H., Roman, C.A.F., Ukueberuwa, D.M., Shapiro, M.D., \& Arnett, P.A. (2017). Normative data for a comprehensive neuropsychological test battery used in the assessment of sports-related concussion. Archives of Clinical Neuropsychology: The Official Journal of the National Academy of Neuropsychologists, 32(2), 168-183. doi: 10.1093/arclin/acw090.

Mohn, C. \& Rund, B.R. (2016). Neurocognitive profile in major depressive disorders: Relationship to symptom level and subjective memory complaints. BMC Psychiatry, 16. doi: 10.1186/ s12888-016-0815-8.

Moser, R.S., Iverson, G.L., Echemendia, R.J., Lovell, M.R., Schatz, P., Webbe, F.M., Ruff, R.M., \& Barth, J.T. (2007). Neuropsychological evaluation in the diagnosis and management of sports-related concussion. Archives of Clinical Neuropsychology, 22(8), 909-916. doi: 10.1016/j.acn.2007.09.004.

Paulus, D.J., Vanwoerden, S., Norton, P.J., \& Sharp, C. (2016). From neuroticism to anxiety: Examining unique contributions of three transdiagnostic vulnerability factors. Personality and Individual Differences, 94, 38-43. doi: 10.1016/j.paid.2016.01. 012.

Peterson, R.A. \& Heilbronner, R.L. (1987). The anxiety sensitivity index: Journal of Anxiety Disorders, 1(2), 117-121. doi: 10.1016/ 0887-6185(87)90002-8.

Pratt, L.A., Druss, B.G., Manderscheid, R.W., \& Walker, E.R. (2016). Excess mortality due to depression and anxiety in the United States: Results from a nationally representative survey. General Hospital Psychiatry, 39, 39-45. doi: 10.1016/j. genhosppsych.2015.12.003.

Rabinowitz, A.R. \& Arnett, P.A. (2013). Intraindividual cognitive variability before and after sports-related concussion. Neuropsychology, 27(4), 481-490. doi: 10.1037/a0033023.

Reiss, S., Peterson, R.A., Gursky, D.M., \& McNally, R.J. (1986). Anxiety sensitivity, anxiety frequency and the prediction of fearfulness. Behaviour Research and Therapy, 24(1), 1-8. doi: 10. 1016/0005-7967(86)90143-9. 
Reynolds, C.R. (2002). Comprehensive Trail Making Test (CTMT). Austin, TX: Pro-Ed.

Riegler, K.E., Guty, E.T., \& Arnett, P.A. (2019a). Validity of the ImPACT Post-Concussion Symptom Scale (PCSS) affective symptom cluster as a screener for depression in collegiate athletes. Archives of Clinical Neuropsychology: The Official Journal of the National Academy of Neuropsychologists, 34(4), 563-574. doi: 10.1093/arclin/acy081.

Riegler, K.E., Guty, E.T., \& Arnett, P.A. (2019b). Neuropsychological test performance in depressed and nondepressed collegiate athletes following concussion. Neuropsychology. doi: 10.1037/neu 0000582.

Rodriguez, B.F., Bruce, S.E., Pagano, M.E., Spencer, M.A., \& Keller, M.B. (2004). Factor structure and stability of the Anxiety Sensitivity Index in a longitudinal study of anxiety disorder patients. Behaviour Research and Therapy, 42(1), 79-91. doi: 10.1016/S0005-7967(03)00074-3.
Rosellini, A.J. \& Brown, T.A. (2011). The NEO five-factor inventory: latent structure and relationships with dimensions of anxiety and depressive disorders in a large clinical sample. Assessment, 18(1), 27-38. doi: 10.1177/1073191110382848.

Sandin, B., Chorot, P., \& McNally, R.J. (2001). Anxiety sensitivity index: normative data and its differentiation from trait anxiety. Behaviour Research and Therapy, 39(2), 213-219. doi: 10.1016/ S0005-7967(00)00009-7.

Smith, A. (1991). Symbol Digit Modalities Test. Los Angeles, CA: Western Psychological Services.

Trenerry, M.R., Crosson, B., DeBoe, J., \& Leber, W.R. (1989). Stroop Neuropsychological Screening Test. Odessa, FL: Psychological Assessment Resource.

Weschler, D. (1997). Weschler Adult Intelligence Scale-III New York, NY: Psychological Corporation.

Widiger, T.A. (2011). Personality and psychopathology. World Psychiatry, 10(2), 103-106. 